\title{
Automatic Inspection of Spot Welds by Thermography
}

Anna Runnemalm, Jörgen Ahlberg, Anders Appelgren and Stefan Sjokvist

\section{Linköping University Post Print}

\section{Tweet}

N.B.: When citing this work, cite the original article.

The original publication is available at www.springerlink.com:

Anna Runnemalm, Jörgen Ahlberg, Anders Appelgren and Stefan Sjokvist, Automatic Inspection of Spot Welds by Thermography, 2014, Journal of nondestructive evaluation, (33), 3, 398-406.

http://dx.doi.org/10.1007/s10921-014-0233-0

Copyright: Springer Verlag (Germany) http://www.springerlink.com/?MUD=MP

Postprint available at: Linköping University Electronic Press http://urn.kb.se/resolve?urn=urn:nbn:se:liu:diva-110472 


\title{
Automatic inspection of spot welds by thermography
}

\author{
Anna Runnemalm • Jörgen Ahlberg • \\ Anders Appelgren • Stefan Sjökvist
}

Received: date / Accepted: date

\begin{abstract}
The interest for thermography as a method for spot weld inspection has increased during the last years since it is a full-field method suitable for automatic inspection. Thermography systems can be developed in different ways, with different physical setups, excitation sources, and image analysis algorithms. In this paper we suggest a single-sided setup of a thermography system using a flash lamp as excitation source. The analysis algorithm aims to find the spatial region in the acquired images corresponding to the successfully welded area, i.e., the nugget size. Experiments show that the system is able to detect spot welds, measure the nugget diameter, and based on the information also separate a spot weld from a stick weld. The system is capable to inspect more than four spot welds per minute, and has potential for an automatic non-destructive system for spot weld inspection. The development opportunities are significant, since the algorithm used in the initial analysis is rather simplified. Moreover, further evaluation of alternative excitation sources can potentially improve the performance.
\end{abstract}

Keywords Spot welds · inspection · non-destructive testing · thermography

Anna Runnemalm

Division of Engineering Science, University West, 46186 Trollhättan, Sweden

Tel.: +46-520-223487

Fax: +46-520-223099

E-mail: anna.runnemalm@hv.se

Jörgen Ahlberg

Dept. of Electrical Engineering, Linköping University, 58183 Linköping, Sweden

Tel. +46-706-757384

E-mail: jorgen.ahlberg@liu.se

Anders Appelgren

Division of Engineering Science, University West, 46186 Trollhättan, Sweden

Tel.: +46-520-223311

Fax: +46-520-223099

E-mail: anders.appelgren@hv.se

Stefan Sjökvist

Termisk Systemteknik AB, Diskettgatan 11B, 58335 Linköping, Sweden

Tel. +46-705-358727

E-mail: stefan.sjokvist@termisk.se 


\section{Introduction}

There is an ever increasing need for efficient quality control in the automotive and aerospace industries, for several reasons. One such reason is that reducing the weight of the manufactured structures as a means of reducing fuel consumption and material costs has high priority. Reduced structural weight results in decreased margins with respect to the structural strength, which leads to an increased need for product quality control. Another reason for quality control is the safety aspects.

With a higher demand for quality control follows a need for general inspection of all manufactured products (not only a sparsely sampled subset), which requires automatic inspection cells in order to be time and cost effective. In addition, the car bodies are today often made of high strength steel and traditional test methods are not permitted. Therefore there is an increased need of new developed non-destructive testing methods suited for automatic inspection.

In order to meet these demands, systems for for non-destructive testing (NDT) using thermography (thermal non-destructive testing, TNDT) has met an increased interest. TNDT systems can be developed in different ways depending on the application. Different arrangements requires different setups, excitation sources, and image analysis algorithms. Examples of different setups include singlesided and double-sided (transmission) setups, and possible excitation sources include ultrasound, flash lamps, and induction heating.

Aiming for a TNDT method suitable for use in automotive production, there are three key aspects that need to be considered. First, the method needs to give robust and reliable results. Second, since not all joints allow for double-sided access, the method has to be single-sided. Third, in order to be able to inspect a high number of spot welds in a running production line, the method needs to be fast. In this paper we propose a single-sided set up of a thermography system for spot weld inspection. The set-up is arranged to be possible to mount on an industrial robot in order to achieve a fully automatic inspection system. The analysis algorithm aims to find the spatial region in the acquired images that corresponds to the successfully welded area (the nugget size). The presented system is capable to inspect more than four spot welds per minute.

\subsection{Related work}

Thermography has been reported as a method for NDT in general $[1,2]$. In recent years it has also been presented as a non-destructive testing method for joints, see e.g. [4], and specifically for spot welds, see e.g. [5-8]. The interest for this method has increased during the last years, since it is a full-field method suitable for automatic inspection.

Woo et al. [5] suggested different excitation techniques, e.g. flash lamp and ice cubes. All experiments were conducted with a transmission set-up, double sided. Both Schlichting et al. [7] and Shpartko et al. [8] used a flash lamp for heating a spot weld area and determined the spot weld position and size in a transmission setup. Lee et al. [6] used lock-in thermography to measure the nugget size of a spot weld. They used a single-side experimental set up, and compared ultrasoundinfrared, photo-infrared, and lock-in thermography methods. Their conclusion was that the lock-in method was the most suitable for nugget sizing. However, lock-in 
thermography has one major disadvantage when it comes to automation; it is more time consuming than pulsed thermography which is used in this paper.

\subsection{Outline}

The outline of this paper is as follows. In Section 2, resistance spot welding and the inspection (destructive and non-destructive) of spot welds are introduced. The experimental setup used is presented in Section 3, and the proposed image analysis algorithm in Section 4. The results are described in Section 5, and conclusions are drawn in Section 6.

\section{Spot welds and inspection}

Resistive spot welding (RSW) [9] is one of the most common methods in automotive industries to join metal sheets. The principle of RSW is that two electrodes clamp two or more metal sheets together. Forcing a large electric current through the electrodes (and thus the metal sheets), the metal between the electrodes will melt and a weld nugget is formed when the metal has cooled down sufficiently. Then the electrodes release the nugget. An attractive feature of RSW is that a large amount energy can be delivered to the spot in a very short time (fractions of a second). That permits the welding to occur without excessive heating of the remainder of the sheet. The process is illustrated in Fig. 1.

\subsection{Quality control of spot welds}

A measure of the quality of a spot weld is the diameter of the weld nugget since it directly relates to the strength. Quality control can be either destructive or non-destructive. Destructive testing is still the most common method to test spot welds. There are two standardized destructive testing methods; peel and chisel test [10]. However, these methods are time consuming, costly, and unfeasible in running production.

Most non-destructive testing methods used for fusion welds can also be used on spot welds. In the automotive industry, the most common method for inspection of spot welds is ultrasonic testing, but experience shows that the reliability of the test is often depending on the time, place, testing situation, and skill of the operator. With the suggested setup of an automated thermography system, the inspection can be operator-independent, and with a stationary inspection cell, as suggested here, the time, test place and situation can be controlled.

\subsection{Thermography}

The principle of detecting or characterizing a defect or feature in a material using TNDT is shown in Fig. 2. The following components can be identified:

- Test piece with a possible defect or other feature, such as a spot weld.

- Excitation source (heat source). 


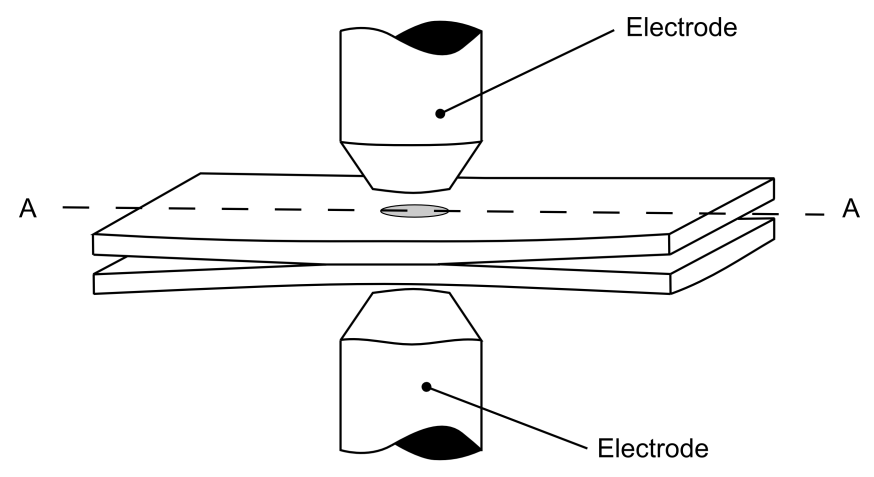

A-A

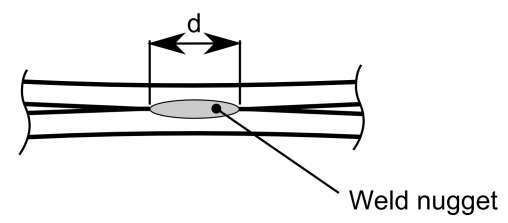

Fig. 1 The principle of resistive spot welding. The diameter, d, of the weld nugget is a quality measure of the spot weld.

- Thermal camera to record the surface temperature of the test piece as a function of time.

- Computer for analysis, equipped with algorithms specially designed for the task.

Thermal energy, generated from a heat source, excite a test piece. The heat at the surface of the test piece will vary depending on time, heat source, material, defects, and surface variations in the test piece. A thermal camera registers the thermal distribution at the surface of the test piece. By analyzing this spatial heat distribution, information about internal variation of heat conductivity of the test piece (resulting from e.g. cracks) can be extracted.

In order to be able to detect and/or characterize a defect or feature in the test piece, it is important to consider the properties of the studied object and carefully design the components of the TNDT system. In the particular case of spot weld inspection, the heat induced at the surface of the test sample is assumed to dissipate through the weld nugget quickly compared to the surrounding area.

The heat source, camera, and algorithm used in this study are described in the next chapter.

\section{Experimental setup}

A TNDT system for spot weld inspection was assembled as shown in Fig. 3. A thermal camera (FLIR SC5650) with a $(N \times M) 640 \times 512$ pixel detector area made of InSb and a band width of $2.5-5.1 \mu \mathrm{m}$ was used. The frame rate of the camera was 100 frames per second, and the original $27 \mathrm{~mm}$ optical lens was used. Since image data will be normalized in a later stage (see Section 4), recording can 

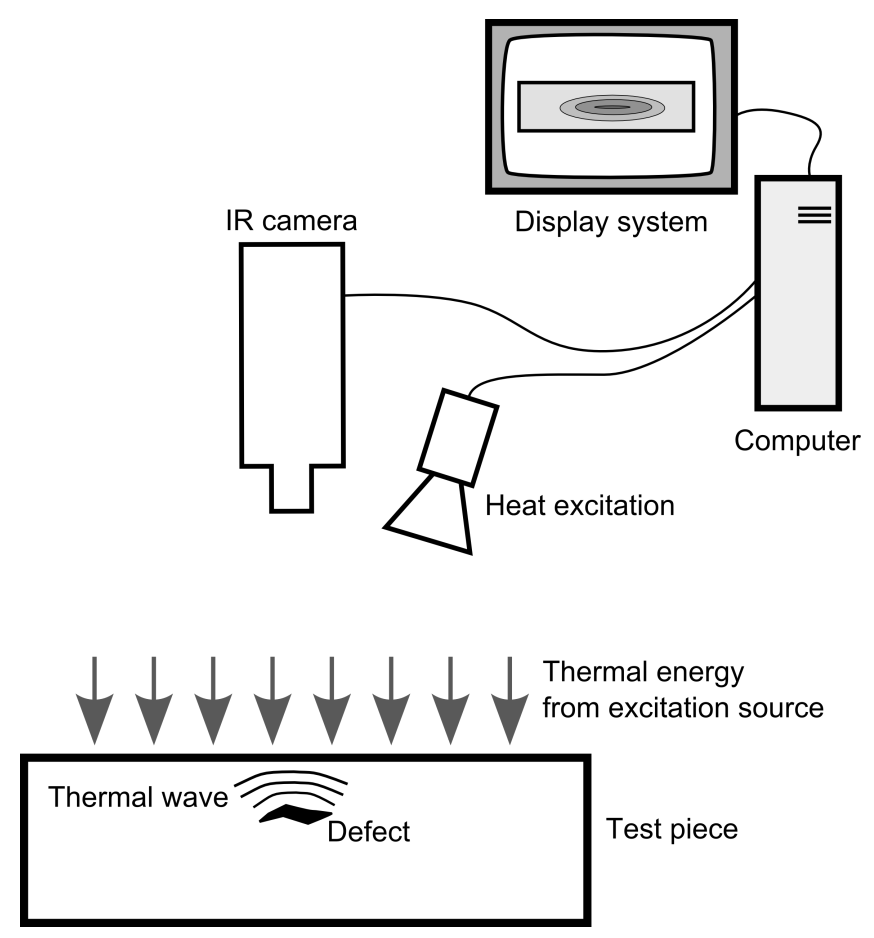

Fig. 2 A general system for thermal non-destructive testing (TNDT).

be done either in terms of raw signals or interpreted as temperature. In this paper the raw data was used.

As excitation (heat) source, a flash lamp (Hensel EH PRO 6000) together with a flash generator (Hensel $6000 \mathrm{~S}$ ) was used. Nominally, a $6 \mathrm{~kJ}$ flash during 0.04 seconds is achieved with this heat source.

The test samples used for evaluation in this paper were spot welded metal sheets in two test series, I and II. The material in the metal sheets were Boron steel and Zinc coated mild steel with the material thickness of $1.5 \mathrm{~mm}$ and 0.8 $\mathrm{mm}$ according to Table 1 . The spot welds all had a desired nugget size of $6 \mathrm{~mm}$. The default parameters for the spot welds were according to Table 2. To achieve different nugget sizes and also stick welds the weld current was varied from $5.5 \mathrm{kA}$ to $9 \mathrm{kA}$ for test series I and from $7 \mathrm{kA}$ to $11 \mathrm{kA}$ on test series II.

The thermal camera and the flash lamp were mounted on the same side of the test plates, since a single-sided inspection system is required in a production line. To reduce disturbances from reflections, the thermal camera and the flash lamp were mounted with an angle relative the normal of the test piece. The angle to the thermal camera, $\alpha$, was approximately 5 degrees, and to the flash lamp, $\beta, 35$ degrees. The distance between the thermal camera and the test piece was $260 \mathrm{~mm}$. The positioning of the thermal camera and the flash lamp was selected to avoid direct reflection in order to reduce noise in the measurement signal.

When performing the experiments, the flash lamp heated the surface of the test piece with a $0.04 \mathrm{~s}$ long light flash. The camera recorded the thermal radiation from the test piece during two seconds, starting slightly before the flash. 


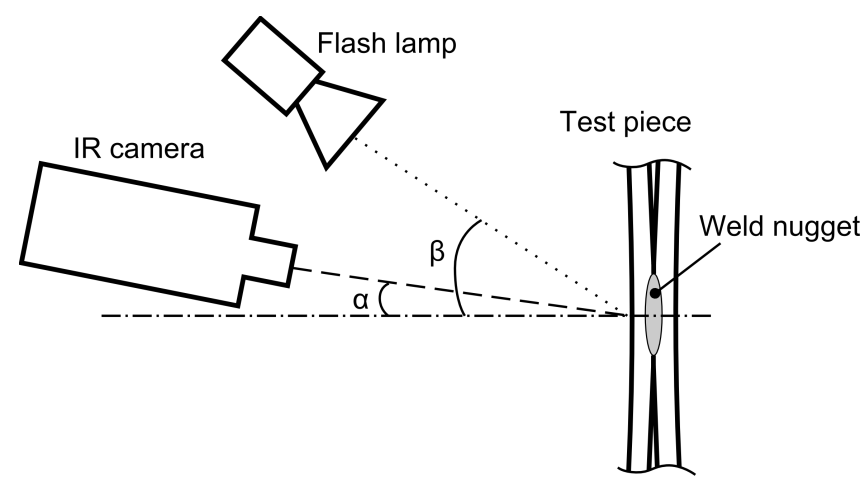

Fig. 3 Our experimental setup for inspection of a spot weld using thermography. The camera and the flash lamp are angled relative the normal to the surface of the test piece.

Table 1 Test pieces of spot welds for experimental evaluation. Test plates are made of two metal sheets, A and B, with different materials and thicknesses.

\begin{tabular}{ccccc}
\hline $\begin{array}{c}\text { Test } \\
\text { serie }\end{array}$ & $\begin{array}{c}\text { Materials } \\
\text { A/B }\end{array}$ & $\begin{array}{c}\text { Thickness } \\
\text { A/B }[\mathbf{m m}]\end{array}$ & $\begin{array}{c}\text { Desired spot } \\
\text { weld [mm] }\end{array}$ & $\begin{array}{c}\text { No. of } \\
\text { plates }\end{array}$ \\
\hline I & Bore steel/Bore steel & $1.5 / 1.5$ & 6 & 5 \\
II & Zinc coated mild steel/Bore steel & $0.8 / 1.5$ & 6 & 5 \\
\hline
\end{tabular}

Table 2 Default weled parameters for the spot welds used in the experiments.

\begin{tabular}{cccccccc}
\hline $\begin{array}{c}\text { Test } \\
\text { serie }\end{array}$ & $\begin{array}{c}\text { Force } \\
{[\mathbf{k N}]}\end{array}$ & $\begin{array}{c}\text { Current } \\
{[\mathbf{k A}]}\end{array}$ & $\begin{array}{c}\text { Weld } \\
\text { time } \\
{[\mathbf{m s}]}\end{array}$ & $\begin{array}{c}\text { Hold } \\
\text { time } \\
{[\mathbf{m s}]}\end{array}$ & $\begin{array}{c}\text { Electrode } \\
\text { size } \\
{[\mathbf{m m}]}\end{array}$ & $\begin{array}{c}\text { Contact } \\
\text { diameter } \\
{[\mathbf{m m}]}\end{array}$ & $\begin{array}{c}\text { Electrode } \\
\text { radius } \\
{[\mathbf{m m}]}\end{array}$ \\
\hline I & 4.6 & 6.5 & 400 & 200 & 20 & 8 & 50 \\
II & 2.4 & 9 & 310 & 130 & 20 & 8 & 50 \\
\hline
\end{tabular}

\section{Analysis algorithm}

Recall from Section 2.2 that the underlying assumption is that the heat induced at the surface of the test piece dissipates through the weld nugget quickly compared to the surrounding area. Thus, finding the nugget shape and size in the the thermal image boils down to finding a region that has a faster cooling rate than the neighborhood. Under ideal circumstances, such as having a noise-free sensor and a test piece with uniform and high emissivity, the problem would be trivial (simply find the dark region in one of the thermal images). In reality, a more elaborate analysis method is needed due to non-ideal situation.

The proposed processing chain contains six steps, as follows:

1. Find the flash position (in time) in the sequence of images.

2. Normalize the images.

3. Select a reference image.

4. Find the position of the weld nugget.

5. Extract the shape of the nugget.

6. Post-process the found shape and decide the quality of the nugget. 
The input to the first step is the sequence of images acquired by the thermal camera. The output from the last step is the estimated dimensions of the weld nugget. The processing steps are explained in the following sections.

\subsection{Finding the flash}

Since the camera is not triggered by the flash but records continuously, the time instant of the flash needs to be found. This is easily done by finding the image that has the largest sum of pixel values. The following $K$ images, that is, the ones that show the cooling process, are selected for further processing. The value of $K$ needs to be sufficiently large to allow the temperature in the test piece to reach a steady state. In practice, a one or a few seconds of image data is enough. With the frame rate of $100 \mathrm{~Hz}$ that was used in the experiments, that corresponds to a value of $K$ of a one or a few hundred (we used values of $K$ in the range 100-250). The selected images are kept in a data cube; an $N \times M \times K$ matrix $\mathbf{A}$, where $N \times M$ corresponds to the camera resolution stated above.

Below, we write $\mathbf{a}_{k}$ for the $k$ :th image and $a_{x y k}$ is the pixel intensity value at image coordinate $(x, y)$ in $\mathbf{a}_{k}$.

\subsection{Normalization.}

In order to compensate for varying emissivity across the test piece's surface, the image data is normalized so that the sequence of measured values are between one and zero for each pixel. The normalized data cube $\mathbf{B}$ thus have the pixel values

$$
b_{x y k}=\frac{a_{x y k} a_{x y K}}{a_{x y 1} a_{x y K}}
$$

That is, the temperature curve for each pixel, such as the ones in Fig. 6, will start at the value one (just after the flash) and eventually reach zero (steady state).

\subsection{Reference image selection}

We select a reference image $\mathbf{r}=\mathbf{b}_{j}$ on which we will perform spatial analysis i.e. find the nugget region. The image should be selected from a point in time where the surface temperature difference between nugget and non-nugget is as large as possible. This timing is not critical; values of $j$ in the range $25-50$ (i.e., a time of $250-500 \mu \mathrm{s}$ after the flash) have been used without observable performance differences in this study.

\subsection{Finding the weld nugget position}

The position of the spot weld in the real world coordinate system is known, and the position and angle of the camera is known with some precision depending on the robot on which the camera is mounted. Thus one can assume that the spot weld is positioned near the center of the image, and search for the spot weld within 
a relatively small area (a few centimeters wide) simply by finding the minimum value in a spatially low-pass filtered version of the image, that is

$$
\left(x^{*}, y^{*}\right)=\arg \min _{x, y}(\mathbf{h} * \mathbf{r})_{x y},
$$

where $\mathbf{h}$ is a suitable filter kernel and $*$ denotes convolution. In the experiments a $9 \times 9$ averaging kernel was used.

\subsection{Extracting the shape of the nugget}

In order to segment the colder/darker area of the nugget, we search for a Maximally Stable Extremal Region (MSER, [11]) encompassing the pixel coordinate $\left(x^{*}, y^{*}\right)$ in the image $\mathbf{r}$. An extremal region is defined as the region given by thresholding the image, i.e., the collection of pixels satisfying $r_{x y}<t$ for some threshold $t$. The extremal region is said to be stable if its area does not change much when varying the threshold. The maximally stable extremal region is the region that changes the least for some interval of $t$.

The process to extract the MSER goes as follows. First, we threshold the image $\mathbf{r}$ using several different thresholds, resulting in binary images $\mathbf{b}(t)$ with pixel values

$$
b_{x y}(t)=\left\{\begin{array}{l}
1 \text { if } r_{x y}<t \\
0 \text { otherwise }
\end{array} .\right.
$$

In our experiments, we vary $t$ in the range

$$
\left[r_{x^{*} y^{*}}, \frac{1}{2}\left(r_{x^{*} y^{*}}+\max _{x, y} r_{x y}\right)\right]
$$

in steps of 0.001. We label the connected components in each binary image, and remove redundant components not encompassing $\left(x^{*}, y^{*}\right)$, creating new binary images $\mathbf{b}^{\prime}(t)$. In each such image, there will be a number of 1 's corresponding to the number of pixels in the extremal region. By counting the 1's, i.e., measure the area of the extremal region, we get a function

$$
n(t)=\sum_{x y} b_{x y}^{\prime}(t)
$$

Minimizing the differential of $n(t)$ with respect to the threshold, the MSER is given by the binary image $\mathbf{b}^{\prime}\left(t^{*}\right)$, where

$$
t^{*}=\arg \min _{t} \frac{d n(t)}{d t}
$$

This differential is estimated by using a 13-point filter.

In order to reduce sensitivity to noise, extremal regions were found in $\mathbf{r}$ as well as in two low-passed filtered versions of $\mathbf{r}$, in practice forming a scale hierarchy. The maximally stable extremal region from these three images is then selected. 
4.6 Post processing

The border of the region extracted above is typically quite noisy, and the region might also contain holes. This is due to the fact that each pixel is thresholded separately from its neighbors. A final morphological closing (see e.g. [12]) using a disc-shaped structuring element of size 13 is done in order to fill holes and notches in the shape. Such closing is illustrated in Fig. 4.

The final output is illustrated in Fig. 6, where also the cooling curves for four reference points are shown. The width and height of the extracted shape is measured, and, for the purposes of evaluation (see Section 5.1), averaged.

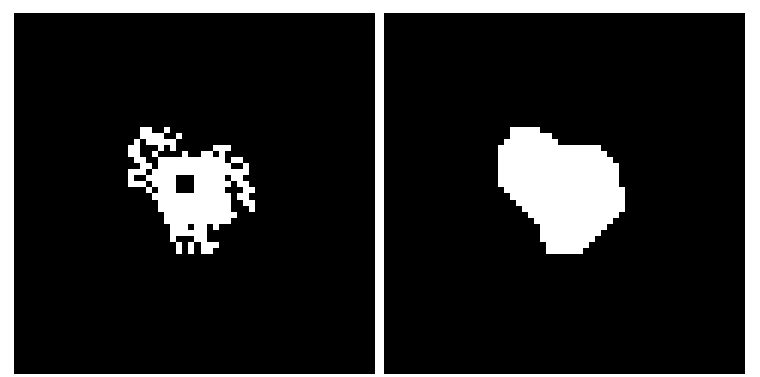

Fig. 4 A synthetic (not showing a real nugget shape) example showing the effect of morphological closing.

\section{Experiments}

A series of experiments was conducted in order to evaluate the analysis algorithm. Ten test samples, each consisting of a spot welded two-plates combination, were measured. The test was divided into two test series, see Table 1 . The first test series (I) contained five test samples made of Boron steel with equal plate thickness, 1.5 $\mathrm{mm}$, see Table 1. The second test serie (II) contained of another five samples made of sheet metals with different thicknesses; 1.5 and $0.8 \mathrm{~mm}$. The $1.5 \mathrm{~mm}$ thick plates were made of Boron steel, and the $0.8 \mathrm{~mm}$ plates of Zinc coated mild steel. The spot welds were produced with the aim to reach a diameter of $6 \mathrm{~mm}$. Two test samples (II.1 and II.5) with stick welds were included.

The different metal sheets have different emissivity. Zinc coated mild steel has a low emissivity, and thus high reflectivity and low absorptivity. For thermography inspection on such materials, more measurement noise will be introduced, due to reflection of infrared radiation from hot objects in the surrounding, including the excitation source. In addition, low absorptivity leads to lower heating of the sample if a flash lamp is used as excitation source. By positioning the measurement system as in Fig. 3, the direct reflections may be avoided and the problem with high reflectivity thus be reduced. Second, by using a thermal camera with high sensitivity, the relative low change of temperature in the test sample will not be a problem for the measurement. Different materials with varying emissivity across the surface can lead to problems if the following analysis is based on the actual 


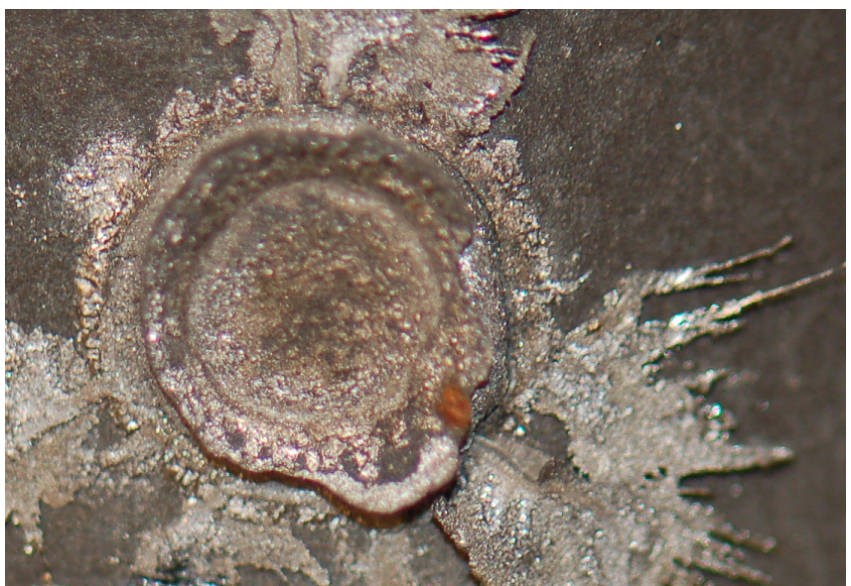

Fig. 5 A test piece with expulsion from the spot welding (after peel test).

measured temperature. In this paper, the analysis is based on the variation in measured infrared radiation with respect to time. Therefore the absolute temperature, which require exact knowledge about the emissivity, is not needed.

All test pieces where measured and analyzed with the TNDT system from both sides, i.e., each test piece gave two measurement samples. The results were compared to the results from a peel test for each piece. In order to avoid uncertainties in the comparative measurement, the peel tests were performed by a skilled person with experience of measuring spot weld diameters. During the peel test, the two plates were peeled apart using a wedge. The measured spot weld diameter is the average of the maximum and the minimum diameter of the plug. For a more detailed explanation on how to perform a peel test, see [10].

In addition to the test series above, a few spot welds with expulsions were measured with the TNDT system in order to evaluate the possibility for the system to detect expulsions. A test sample with expulsion from the spot weld can be seen in Fig. 5, after peel test.

\subsection{Results}

Results from the TNDT system is exemplified in Fig. 6. The vertical and horizontal diameter of the estimated weld nugget are measured in pixels, and easily transformed to millimeters by using information of the camera's resolution, field of view, and distance to the test piece.

In Table 3, the measured diameters of the spot welds are given, measured by the TNDT system and by peel tests. The measurements from the TNDT system are conducted from both sides of the test samples (indicated by a and b). The results for the various test pieces are discussed below:

- Bore steel. As can be seen in Table 3, the results from the two sides are almost equal, except from plate I.2 (I.2a in comparison to I.2b), indicating that the method is stable. However, the measured diameter with the TNDT system is in general slightly smaller compared to the the peel test measurement. Further 
tests are needed in order to determine if this is a systematic bias, which could then be compensated for.

- Zinc coating. The measurements on zinc coated mild steel (series II, side a) were disturbed. This is due to reflection in the shiny surface of the plate, and can be avoided by a slightly different experimental setup (different angles of flash lamp and camera), by using another type of excitation source (such as induction heating), or by covering the measurement area with some highemissivity material (e.g., powder).

- Stick welds. As mentioned, two test pieces with stick welds were included (II.1 and II.5). In those pieces, the TNDT system was not able to find any spot welds, which indicates that the system is able to distinguish a spot weld from a stick weld.

- Partial stick welds. The final output of the analysis algorithm, as reported in Table 3, is the average of two perpendicular distances (width and height) of the measured spot weld. If the spot weld is non-uniform, as is the case for a partial stick weld, the result will be a radius smaller than the largest dimension of the true spot weld.

- Expulsions. Results from the additional measurements on spot welds with expulsion can be seen in Fig. 8. The system could not distinguish between these welds and welds without expulsion, and, as a consequence, the estimated contours are irregular and the diameters exaggerated by the TNDT system. This is due to the physical contact between the two plates by the expulsion, resulting in heat conduction between the plates, as in the spot weld. With an improved analysis algorithm, this could probably be handled, since the heat conduction is different through an expulsion compared to through a spot weld.

Even if the extracted characteristics of the welds most of the times are correct, the analysis is sometimes confused by the physical deformations of the surface created by the welding process, see for example Fig. 7 where the indentation of the spot weld has resulted in disturbances in the measurement. As mentioned, we might also have a bias towards somewhat smaller nuggets. This bias is presumably dependent on the thickness and heat conductivity of the material, and needs to be investigated in the future.

\section{Conclusion and future work}

We have developed and tested a TNDT system for single-sided inspection of spot welds. Assuming that the weld nugget will conduct heat away from the surface faster than its surroundings, we have shown that the extraction of Maximally Stable Extremal Regions (MSERs) in a thermal image can reveal the nugget size and shape. The results show that the system is able to detect spot welds, measure the diameter, and separate a spot weld from a stick weld. In conclusion, the system is shown to have potential for an automatic system for spot weld inspection.

There are remaining challenges related to the surface properties of the test samples. First, shiny surfaces can be a problem due to reflections from the heat source used. This can potentially be solved in various ways, and needs further investigation. Another challenge is weld expulsions, as they make the spot welds look larger and more irregular.

Additionally, there are several issues still to be investigated in the future: 

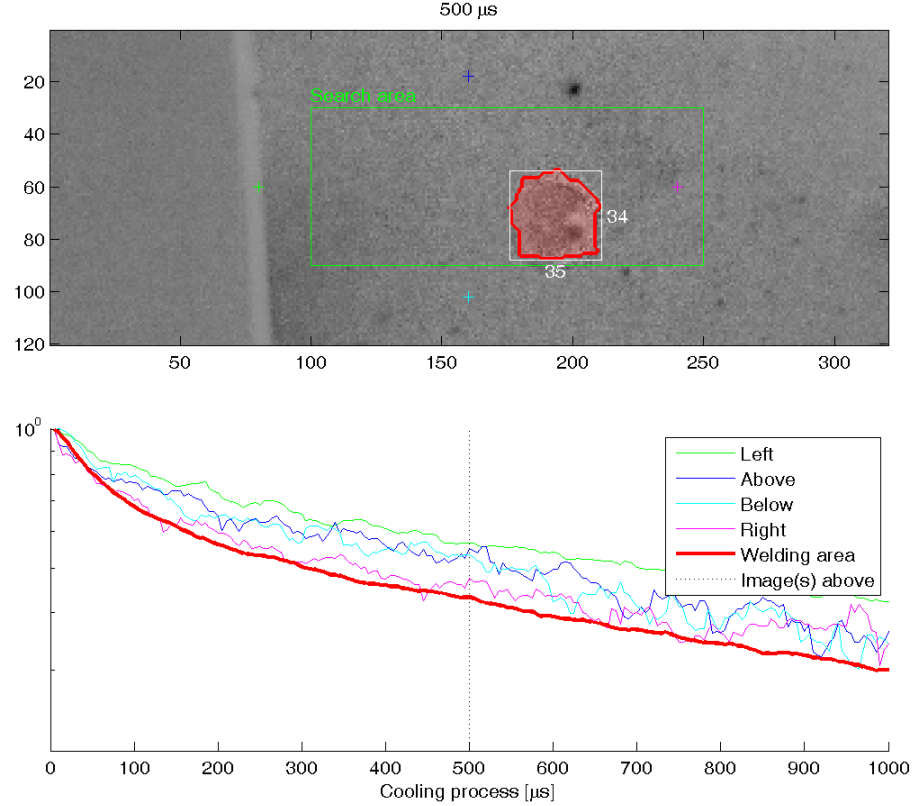

Fig. 6 Top: The image half a second after the flash is shown (chosen for visualization). Spot welds are searched for within the green rectangle, and the red shape marks the estimated weld area. The enclosing rectangle (white) and its horizontal and vertical extension (measured in pixels) is shown as well. Bottom: The cooling process in the normalized sequences corresponding to the small crosses in the top image and the mean over the welding area. The normalization means that each pixel cools from an initial value of one (immediately after the flash) to zero (approximately at steady state). Note that the y-axis has logarithmic scale, i.e., the final value of zero cannot be plotted.

- Bias as a function of material properties. The currently implemented system seems to give a bias towards smaller spot weld sizes. This bias is probably dependent on the material properties (heat conductivity and thickness) and should be systematically studied.

- Image analysis. The current implemented image analysis method has several limitations. A more general implementation, where physical properties are taken into account, preferably validated by theoretical calculations and/or simulations, would be more reliable. Also, the method does not exploit the expected shape characteristics of the spot welds - the erroneous result shown in Fig. 7 could be avoided by exploiting the visibly circular structure of the weld.

- Simulations. The problem with peel tests is that they are time-consuming and expensive to perform in large numbers. In order to evaluate variations and enable the work suggested in the previous two items, a simulation model should be built and used for large scale tests. Real peel tests could then be used in a small number in order to validate the model.

- Excitation source. During the study several questions regarding the excitation were raised. The influence of the heat from two adjacent flashes should be examined, and shiny surfaces need to be handled. Moreover, for a system to be used in a production plant, the influence on the work environment is important. 

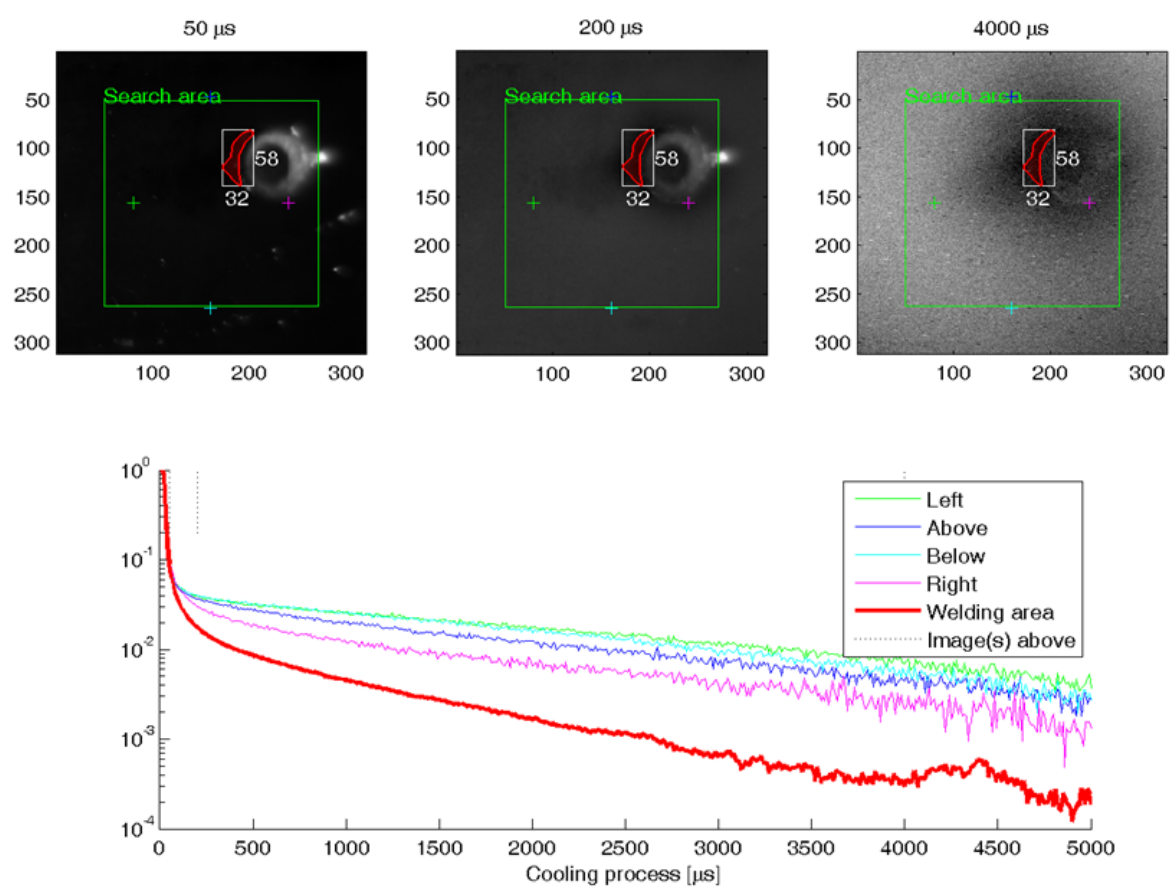

Fig. 7 Image analysis as in Fig. 6, but the analysis method lock on a spot on the outside of the welding mark.
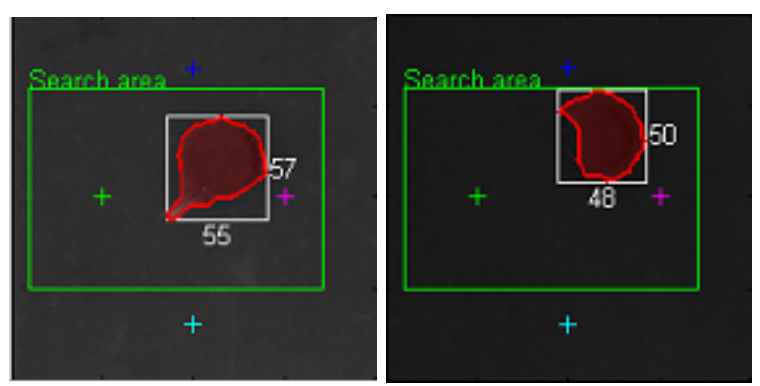

Fig. 8 Examples of results from thermography measurements on spot welds with expulsion. The estimated contour of the spot weld is often irregular and its size larger compared to what is given by a peel test.

Acknowledgements The work originated from the research project SpotLight, funded by Vinnova (the FFI program). SpotLight was carried out in cooperation with Saab Automobile AB, Scania, Gestamp Hardtech, SSAB, SAPA, SWEREA IVF, Swerea KIMAB, Volvo Cars, and Volvo Trucks.

\section{References}

1. Shull, P. J.: Nondestructive evaluation: theory, techniques and applications. Marcel Dekker, New York (2002). 
Table 3 Results of spot weld diameter measurements by the thermography system in comparison to peel tests. Test plates are named according to material combination (test series I or II), test plate ( 1 to 5 ) and measured side with thermography system (a or b).

\begin{tabular}{|c|c|c|c|}
\hline $\begin{array}{l}\text { Test plate } \\
\text { number }\end{array}$ & $\begin{array}{l}\text { Diameter } \\
\text { peel test } \\
{[\mathrm{mm}]}\end{array}$ & $\begin{array}{c}\text { measured by } \\
\text { thermography } \\
{[\mathrm{mm}]}\end{array}$ & Comment \\
\hline $\begin{array}{l}\text { I.1a } \\
\text { I.1b }\end{array}$ & 6,3 & $\begin{array}{l}5,6 \\
5,5\end{array}$ & \\
\hline $\begin{array}{l}\mathrm{I} .2 \mathrm{a} \\
\mathrm{I} .2 \mathrm{~b}\end{array}$ & 6,0 & $\begin{array}{l}6,4 \\
4,7\end{array}$ & \\
\hline $\begin{array}{l}\mathrm{I} .3 \mathrm{a} \\
\mathrm{I} .3 \mathrm{~b}\end{array}$ & 5,6 & $\begin{array}{l}5,4 \\
5,2\end{array}$ & \\
\hline $\begin{array}{l}\mathrm{I} .4 \mathrm{a} \\
\mathrm{I} .4 \mathrm{~b}\end{array}$ & 6,1 & $\begin{array}{l}5,1 \\
4,8\end{array}$ & \\
\hline $\begin{array}{l}\mathrm{I} .5 \mathrm{a} \\
\mathrm{I} .5 \mathrm{~b}\end{array}$ & 6,3 & $\begin{array}{l}5,1 \\
5,0\end{array}$ & \\
\hline $\begin{array}{l}\text { II.1a } \\
\text { II. } 1 \mathrm{~b}\end{array}$ & 0,0 & $\begin{array}{l}0,0 \\
0,0\end{array}$ & Stick weld \\
\hline $\begin{array}{l}\text { II. } 2 \mathrm{a} \\
\text { II. } 2 \mathrm{~b}\end{array}$ & 5,8 & $\begin{array}{c}\mathrm{N} / \mathrm{A} \\
3,6\end{array}$ & Disturbed thermography measurement \\
\hline $\begin{array}{l}\text { II. } 3 \mathrm{a} \\
\text { II. } 3 \mathrm{~b}\end{array}$ & 5,8 & $\begin{array}{c}\mathrm{N} / \mathrm{A} \\
4,5\end{array}$ & Disturbed thermography measurement \\
\hline $\begin{array}{l}\text { II. } 4 \mathrm{a} \\
\text { II. } 4 \mathrm{~b}\end{array}$ & 6,1 & $\begin{array}{c}\mathrm{N} / \mathrm{A} \\
4,8\end{array}$ & Disturbed thermography measurement \\
\hline $\begin{array}{l}\text { II. } 5 \mathrm{a} \\
\text { II. } 5 \mathrm{~b}\end{array}$ & 0,0 & $\begin{array}{l}0,0 \\
0,0\end{array}$ & Stick weld \\
\hline
\end{tabular}

2. Maldague, X. P. V.: Theory and practice of infrared technology for nondestructive testing. Wiley, New York (2001).

3. Hung, Y. Y., Chen, Y. S., Ng, S. P., Liu, L., Huang, Y. H., Luk, B. L., Ip, R. W. L., Wu, C. M. L.: Review and comparison of shearography and active thermography for nondestructive evaluation. Materials Science and Engineering: R: Reports 64(5-6), 73-112 (2009). DOI 10.1016/j.mser.2008.11.001

4. Meola, C., Carlomagno, G. M., Squillance, A., Giorleo, G.: The use of infrared thermography for nondestructive evaluation of joints. Infrared Physics and Technology 46(1-2), 93-99 (2004). DOI 10.1016/j.infrared.2004.03.013

5. Woo, W., Chinc, C. W., Fenga, Z., Wang, H., Zhang, W., Xu, H., Sklada, P. S.: Application of infrared imaging for quality inspection in resistance spot welds. In: SPIE Thermosense XXXI. The International Society for Optical Engineering, Orlando, FL (2009)

6. Lee, S., Nam, J., Hwang, W., Kim, J., Lee, B.: A study on integrity assessment of the resistance spot weld by infrared thermography. Procedia Engineering 10, 1748-1753 (2011). DOI 10.1016/j.proeng.2011.04.291

7. Schlichting, J., Brauser, S., Pepke, L. A., Maierhofer, C., Rethmeier, M., Kreutzbruck, M.: Thermographic testing of spot welds. NDT \& E International 48, 23-29 (2012). DOI 10.1016/j.ndteint.2012.02.003

8. Shpartko, P., Lev, L., Kondratyev, N., Soh, J.: Thermography in manufacturing: nondestructive evaluation of ultrasonic spot welds of automotive battery pack. In: 11th Quantitative InfraRed Thermography. Naples, Italy (2012) 
9. Wenman, K. (2012) Welding processes handbook. Woodhead, Cambridge

10. ISO: ISO 10447:2006 resistance welding peel and chisel testing of resistance spot and projection welds (2006).

11. Matas, J., Chum, O., Urban, M., Pajdla, T.: Robust wide baseline stereo from maximally stable extremal regions. Image Vision and Computing 22(10), 761-767 (2004).

12. Sonka, M., Hlavac, V., Boyle, R.: Image processing, analysis, and machine vision. Brooks/Cole Publishing Company (1999) 